\title{
XXI.
}

\section{Zum Vergil.}

Aen. $\mathbf{X}, 185-88$ :

Non ego te, Ligurum ductor fortissime bello, Transierim, Cinyra, et paucis comitate Cupavo,

Cuius olorinae surgunt de vertice pennae

Crimen amor vestrum - formaeque insigne paternae.

Wer ist führer der Ligurer, Cinyras oder Cupavo? Die herausgeber erklären sich theils für den einen, theils für den anderen, und nehmen darnach Cinyra theils für den vocat., theils für den ablat. Soll Cinyras der führer sein, so ist es auffallend, dass, da durch die worte: Non ego - Transierim die aufmerksamkeit auf ihn gelenkt war, im folgenden nicht von ihm, sondern von Cupavo gesprochen wird, und dieser demnach als hauptperson erscheint. Ferner wäre Verg. uns näberen aufschluss über den Cupavo und sein verhältniss zum Cinyras schuldig geblieben. Denn hätte Verg. den Cinyras und den Cupavo für söhne des Cycnus gehalten und gemeint, beide seien ihrem vater in der herrschaft gefolgt, wie allerdings Forbiger und auch Jacoli in s. mythol. lex. angenommen haben, so hätte er doch bei der wahrscheinlichkeit, von seinen lesern missverstanden zu werden, unmöglich nur einen anreden und diesen den Ligurum fortissimus ductor nennen können; auch hätte der folg. relativsatz nicht ausschliesslich vom Cupavo handeln dürfen. Soll dagegen Cupavo der führer der Ligurer sein, so vermisst man eine nähere angabe über die person des Cinyras, denn so häufig auch die röm. dichter individualisiren, so konnte Verg. doch unmöglich schreiben: Cinyra et paucis comitate Cupavo, wenn er so wenig wie seine leser zu sagen wussten, wer dieser räthselhafte Cinyras sei, der wie ein mann eingeführt wird, der unus innumeri militis instar habet. Was aus diesen erwägungen sattsam hervorgeht, dass in Cinyra eine corruptel steckt, das findet noch eine anderweitige unterstützung durch die feine beobachtung Lachmann's zu Lucret. p. 272., der lehrt, dass die röm. dichter sich in griech. wörtern nur äusserst selten die elision eines langen endvokals 
gestattet haben, und darum an u. st. die worte Cinyra et für corrupt erklärt. Suchen wir unter solchen umständen bei den handschriften rath, so finden wir, dass Cinyra blosse conjectur ist, der Med. hat Cinyrae, der Rom. Cumare, der Gud. Cinere, die übrigen Cinyre, Cynare, Cynire, Cinira, Cyrina, Cinate, Cycne. Macrobius las, wie aus Saturn. V, 15. hervorgeht, Cinire; eine andere lesart war Cunare, wie die Serviana zeigen, in denen die bemerkung beigefügt ist: quidam duci nomen datum tradunt a Cunaro monte, qui in Piceno. Es liegt auf der hand, dass mit all diesen lesarten nichts anzufangen ist, denn wenn durch einige auch das metrische bedenken Lachmann's beseitigt wird, so finden doch alle sachlichen gründe, die gegen Cinyra vorgebracht sind, durch keine dieser lesarten, die alle nur na. mensvariationen darbieten, ihre erledigung. Eine lesart babe ich bisher noch nicht mitgetheilt, die des Goth. tert.: tacite; da diese handschrift jedoch sehr jung ist und sich nicht absehen lässt, wie tacite, wenn so von Verg. geschrieben wäre, zu so seltsamen corruptelen liätte anlass geben können, so ist diese lesart wol nur für eine scharfsinnige conjectur des schreibers jenes cod. zu halten.

Was ist denn nun aber mit dem räthselhaften Cinyras, oder wie er sonst heissen mag, anzufangen? Um es kurz zu sagen, so schrieb Verg. meiner ansicht nach: miser et paucis com. Cupavo; ein grammatiker, freilich lange vor Servius und Macrobius, setzte über miser die griech. übersetzung KINTPE, die, da die folgenden worte: crimen amor vestrum auf die vermuthung führten, im vorhergehenden seien zwei personen genannt, unwissende abschreiber verleitete, in dem unschuldigen leidensgefährten des Cupavo zu erblicken.

Was bedeuten aljer ferner die worte: crimen amor vestrum? Auf wen ist das crimen vestrum zu beziehen? Die alten grammatiker, auch Asper dachten, wie Servius berichtet, an den Phaethon und Cycnus, ohne zu berücksichtigen, dass von beiden erst im folgenden geredet wird und dass es sich hier um die schwanenfedern am helme des Cupavo handelt. Neuere gelehrte dachten an den Cinyras und Cupavo und fabelten von einer tragischen

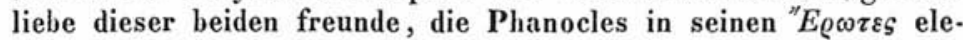
gisch behandelt habe, ohne zu erwägen, wie es komme, dass Servius ganz gegen seine sonstige gewohnheit die schöne gelegenheit, seine mythologischen kenntnisse und seine belesenheit in den alten zu zeigen, unbenutzt gelassen habe und sich hier in ein tiefes stillschweigen hülle. Hätte ein dichter wie Phanocles von dieser liebe etwas gewusst und gesungen, so wäre uns das andenken an den Cinyras doch schwerlich so spurlos untergegangen. Und ganz abgesehen hiervon, so wäre es doch sehr hart und der feinen darstellungsweise Vergil's sehr wenig entsprechend, hier, wo es sich allein vom Cupavo handelt, eine rückbeziehung 
auf den Cinyras einzuflechten. Einen anderen weg schlug Sprengel (neue kritik d. class. dichter p. 139.) ein, dem Rubkopf, Thiel und Jahn in der 1sten ausg. sich angeschlossen haben, indem sie schrieben: crimen, Amor, vestrum und dabei an den Amor und die Venus dachten; doch bedarf diese erklärung, von der sich auch Jahn in der 2ten ausg. losgesagt hat, keiner weiteren widerlegung. Den letzten versuch zur erklärung d. st. machte Wag. ner, dem Forbiger und Süpfle beigetreten sind; Wagner fasst d, st. nämlich als parenthetische apostrophe an die schwanenfedern in dem sinne: ,die liebe, welche Cycnus seinem Phaëthon schenkte, ist die ursache eures ursprungs, d.h. der verwandlung des Cycnus in einen schwan." Freilich ist es das natürlichste, die worte als dichterische anrede an die federn zu nehmen, aber dann kann amor sich unmöglich auf die liebe des Cycnus zum Phaëthon beziehen, sondern kann nur von der liebe der federn selbst verstanden werden, und crimen vestrum kann nicht von der verwandlung in federn, sondern nur von der verwandlung der federn selbst gesagt sein. Wie ich demnach die Wagner'sche erklärung billigen würde, wenn die schwestern des Phaëthon angeredet-würden, so muss ich sie jetzt, da Verg. die schwanenfedern anredet, entschieden verwerfen. Ohne mich länger bei den conjecturen: carmen amor vestrum (Wakef), crimen amore datum (Jahu) und; crinibus ornamentum (Peerlk.) aufzuhalten, erkläre ich mich dahin, dass die fraglichen worte, die jeder annehmbaren erklärung zu spotten scheinen, als späterer versuch, den vergilischen halbvers: Formae insigne paternae zu vervollständigen, zu streichen sind.

In der rede der mutter des Euryalus, in welcher sie den tod ihres sohnes beklagt, sind IX, 483-89:

- nec te, sub tanta pericula missum,

Adfari extremum miserae data copia matri?

$485 \mathrm{Heu}$, terra ignota canibus date praeda Latinis Alitibusque iaces! nec te tua funere mater Produxi, pressive oculos, aut volnera lavi, Veste tegens, tibi quam noctes festina diesque Urguebam et tela curas solabar aniles

zwei änderungen der handschr. überlieferung von den herausgehern vorgenommen. date nämlich in $v$. 485. steht, wie Wagner berichtet, nur in paucis admodum libris, in allen übrigen (also doch auch wahrscheinlich im Medic. Wagner ist hier, wie auch an einigen anderen stellen, die genauere angabe über die lesart der einzelnen codd, schuldig geblieben) data. Nun pflegt Verg. allerdings, wie Wagn. ad Georg. II, 125. (vgl. auch Jahn ad Aen. I, 316.) richtig bemerkt hat, bei wiederholungen desselben wortes nach dazwischen tretender grösserer interpunktion die 
form des wortes und seine stelle im verse zu ändern; doch kann auch ein fall eintreten, wo die regeln der kunst gebieten, das. selbe wort in derselben form und an derselben stelle des verses zu wiederholen, und dieser fall tritt hier ein. Offenbar haben wir hier ein beispiel der musikalisch-malerischen darstellungsweise Vergil's; das eintönige der klagen wird durch den sich durch v. 484-86. hindurchziehenden a-laut trefflich gemalt, und wie könnte das einförmige der klagen besser sinnlich dargestellt werden, als durch den gebrauch derselben form desselben wortes an derselben stelle zweier auf einander folgender verse? Die rücksicht auf den in diesen versen vortönenden a-laut empfiehlt auch die beibehaltung des von allen handschriften gebotenen funera im folgenden verse, das Bembus, Bothe, Wagner, Forbiger, Süpfle und Gossrau in funere geändert haben. Freilich ist tua funera nicht als apposition zu te zu nehmen, auch nicht zu construiren: nec te tegens veste produxi tua funera, dennoch aber möchte es den vorzug verdienen sowohl vor der Jahn'schen änderung: te in tua funera wie vor der kühnen Peerlkamp'schen vermuthung: nec fletu funera. Der weg zur erklärung des handschriftl. funera wird sich ergeben, wenn die richtige erklärung des folgenden tegens gefunden ist. Das partic. hat nur Wagn. zu erklären versucht, indem er sagt, es stehe für et texi. In den quaest. Virg. nämlich stellt Wagn. XXIX, 5. den satz auf, die partic. ständen bisweilen für die verba finita, eine behauptung, die ich, für den Verg. wenigstens, entschieden in abrede nehmen muss. Was nämlich zuvörderst die partic. perf. anlangt, so stehen diese in den von Wagn. angeführten stellen entweder proleptisch, wie Aen. IX, 734: adgnoscunt faciem invisam atque inmania membra Turbati subito Aeneadae, oder sie bringen die in ihnen enthaltene bestimmung in unmittelbare beziehung $\mathrm{zu}$ der gegenwart des schreibenden und stehen also da, wo die rück. sicht auf das verb. finitum ein partic. praes. erwarten liess (s. m. anm. z. Georg. I, 206.), wie Aen. I, 708: Tyrii - Convenere, toris iussi discumbere pictis, oder sie stehen endlich rein adjectivisch, wie Aen. IV, 620: sed cadat ante diem mediaque inhumatus arena. In der letzten von Wagn. angeführten stelle Aen. I, 69: submersas obrue puppes ist der sinn allerdings submerge et obrue, doch ist in dieser satzumwandelung $j a$ auch deutlich ausgesprochen, dass durch das submergere eine dem obruere vorhergeheude handlung bezeichnet wird, das partic. perf. steht also in seiner eigentlichen bedeutung: bedecke die schiffe, nachdem sie versenkt sind. Das partic. praes. aber steht in den von Wagn. beigebrachten stellen Georg. II, 56. Aen. VII, 498. 1X, 286. 525. de conatu. In der verbindung des nomin. des partic. praes. mit simul (s. Aen. X, 856; simul hoc dicens adtollit in aegrum se femur, vgl. XII, 758.) ist das partic. als epexegetischer zusatz zu simul anzusehen; in der verbindung simul 
his dictis dagegen Aen. V, 357. und XI, 827. findet sich die im augusteischen zeitalter aufkommende verbindung des simul mit dem ablat. Das partic. endlich, welches in beschreibungen vorkommt, wie Georg. II, 133: laurus erat; folia haud ullis labentia ventis; Flos ad prima tenax, vgl. Georg. III, 504-5. A. VII, 787. steht nicht für das verb. finit., sondern ist ebenso zu erklären, wie der nomin., der ohne verbum so häufig bei Verg. in beschreibungen vorkommt, vgl. Wagn. z. Aen. IV, 202. So bleibt denn ausser u. st. nur noch Aen. X, 193 übrig. Dort heisst es vom Cycnus: Namque ferunt - Dum canit et maestum Musa solatur amorem, Canentem molli pluma duxisse senectam, Linquentem terras et sidera voce sequentem. Stände hier der infinit. statt der partic., so würden wir nur erfahren, dass Cycnus in einen vogel von grauer farbe verwandelt und dann (d. h. nach erfolgter verwandlung) hoch in die lüfte geflogen sei. Während der zweite theil dieses berichtes unser interesse nicht in anspruch nehmen würde, liesse uns der erste im dunkeln über die frage, in welchen vogel Cycnus verwandelt wurde. Beiden übelständen ist dadurch abgeholfen, dass Verg. die partic. linquentem und sequentem gebrauclit hat, denn nun dient v. 193. zur bezeichnung der eigenschaften des vogels und wir lösen, wenn wir noch die erste hälfte des vorhergehenden verses dazu nehmen, das uns vom dichter/aufgegebene räthsel leicht dahin, dass Cycnus in eineñ schoon verwandelt wurde. Wenn demnach das partic. von Verg. nicht für das verb. finit. verwendet ist; so können an der stelle, von der ich ausgegangen bin, die worte veste tegens sich unmöglich als nähere bestimmung an die vorhergehenden worte volnera lavi anschliessen, sondern weisen auf eine versumsetzung hin, durch welche zugleich der accus. tua funera seine erklärung. findet. Setzt man nämlich v. 487 . hinter v. 489 ., so hat man die gefügige construction: nec te produxi, tua fun. veste tegens. Tua funera ist vom leichnam des Euryalus zu verstehen; auffallend ist dabèi allerdings der plüal.; aber da der plural. corpora öfter von einem leichname steht, wie Ovid. met. II, 326 . vom leichname des Phaëthon: Naides Hesperiae trifida fumantia flamma Corpora dant tumulo, und VIII, 236. von der leiche des Icarus: Hunc miseri tumulo ponentem corpora nati Garrula ramosa prospexit ab ilice perdix, so ist nicht abzusehen, warum nicht auch funera von einem leichnam stehen sollte, und so findet es sich auch bei Stat. Theb. XII, 383., wo die Antigona zur Argeia sagt: mea membra tenes, mea funera (d. i. die mir angehörende leiehe, die leiche meines bruders) plangis ${ }^{1}$ ).

fili 1) leh kann mich von d. st. nicht trennen, ohne der merkwürdigen erklärung des'Servius zu gedenken. Dieser grammatiker nämlich nimmt funera als abgekürzte form des adj. funerea und bemerkt: Apud maiores funereas dicebant eas, ad quas funus perlinebat, ut sororem, matrem, wozu ein anderer grammatiker hinzugefügt hat: vel derivavit veteres se-

Philologux. VII Jahrg. 3. 
Dasselbe heilmittel der versumsetzung habe ich Aen. VII, $691-97$. angewendet:

At Messapus, equum domitor, Neptunia proles,

692 Quem neque fas igni cuiquam nec sternere ferro, Iam pridem resides populos desuetaque bello

Agmina in arma vocat subito, ferrumque retractat.

$695 \mathrm{Hi}$ Fescenninas acies Aequosque Faliscos,

Hi Soractis habent arces Flaviniaque arva,

Et Cimini cum monte lacum lucosque Capenos.

Es ist das verdienst Hofman Peerlkamp's, den fehler d. st. entdeckt zu haben, sehr wahr bemerkt er: Mirum acies et Faliscos Aequos. Hi habent arces, arva, montem, locum, lucos, hoc est habitant. Hi habent acies et Faliscos, non convenit. Atque haec ipsa, habent acies et habent Aequos Faliscos, etiam dissimilem verbo habent significationem assignant. Die richtigkeit dieser bemerkung räumen Peerlk.'s nachfolger ein, doch weiss weder Forb. noch Gossrau einen anderen ausweg, als arces für acies zu proponiren, das sie aber doch auch wieder verwerfen, weil arces im unmittelbar folgenden verse wieder vorkomme. Ich setze v. 695 . nun hinter v. 692. und verändere $\mathrm{Hi}$ in Is. War der vers durch versehen der abschreiber erst einmal hinter v. 694. gerathen, so liegt es auf der hand, wie das hier sinnlose is dem hi weichen kounte. Is aber dient dazu, mit nachdruck das voraufgegangene subject nach einem zwischensatze wieder aufzunehmen, vgl. Aen. IX, 595. Zur empfehlung dieser umstellung mag auch der umstand dienen, dass unten v. 794-96. und 797-802. in ganz gleicher anordnung zuerst die völker und dann ihre wohnsitze angegeben werden.

Dagegen muss ich die versumstellung, welche fast alle herausgeber auf den vorschlag Scaliger's Aen. X, 714-18. vorgenommen haben, durchaus missbilligen. Der kampflustige Mezentius, an den keiner der feinde sich heranwagt, wird hier mit einem eber verglichen, der in's netz getrieben so tobt, dass die jäger ihn nur von ferne beschiessen. Dann heisst es nach der gewöhnlichen anordnung der verse weiter:

culus, ut funeram pro funesta diceret, ut homo scelerus, sicuti scelestus vel scelerosus dicebatur. Etymologisch betrachtet stellt sich der erklärung des Serv. nichts entgegen, denn funerus kann ebenso gut zusammengezogene form aus funereus sein, wie florus aus floreus, eburnus aus eburneus etc. Auch könnte Verg. hier, wo er die multer des Euryalus redend einführt, recht gut dies adj. gebraucht haben, wenn es überhaupt in der älteren sprache vorhanden war, da die röm. frauen an alterthümlichen worten und wortformen am längsten festhielten. Es fragt sich nur, ob sich noch irgendwo eine spur dieses adj. findet. Mir freilich ist keine bekannt, aber sollte es nicht etwa in der form funera irgendwo verkannt und für das subst. gehalten sein? Doch mag nun funera hier subst. oder adj. sein, die oben vorgeschlagene versumsetzung wird sich in beiden fällen als nothwendig herausstellen. 
Ille (näml. der eber) autem inpavidus partis cunctatur in omnis,

715 Dentibus infrendens, et tergo decutit hastas:

Haud aliter, iustae quibus est Mezentius irae,

Non ulli est animus stricto concurrere ferro;

Missilibus longe et vasto clamore lacessunt.

Gossrau allein hat die handschriftliche versordnung, nach der v. 714-15. auf v. 718. folgen, beibehalten, jedoch nur, weil die verse auch in dieser folge einen erträglichen sipn geben, und schliesst seine bemerkung zu d. st. mit den worten: ita turbata sunt verba iam ab antiquissimis temporibus, ut probabile sit Virgilium versus non ita absolvisse, ut ab omni parte probandi fuerint. Und doch ist die von den übrigen herausgebern vorgenom. mene versumstellung durchaus unhaltbar, weil wir sonst eine ganz lästige wiederholung erhielten, denn während bereits v. 711. gesagt war: substitit, der eber steht still (als zeichen der verwunderung und unschlüssigkeit), würde dieser begriff in v. 714. wiederholt werden, und ebenso enthielte v. 715. eine reine wiederholung der worte: infremuitque ferox et inhorruit armos in $\mathbf{v}$. 711. Nach der handschriftlichen versordnung dagegen gehen $\mathbf{v}$. $714-15$. auf den Mezentius; tergum ist dann allerdings ungewöhnlich von dem schilde gesagt, doch darüber s. m. anm. z. d. st. Vielleicht aber ist tergum auch gar nicht einmal von dem schilde, sondern ganz eigentlich von dem rücken des Mez. zu verstehen. Mez. war von kampflust erfüllt mitten in die feindlichen schaaren gedrungen, die lanzen stürmten von allen seiten auf ihn ein, vermochten aber den panzer nicht zu durchdringen, weil sie aus zu grosser ferne (longe, v. 718.) geschleudert wurden.

Neustrelitz.

Th. Ladewig.

\section{Vermischtes.}

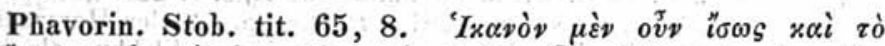

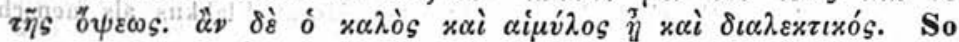
AB. vulg. $\lambda \varepsilon \times \tau \iota x o ́$ s. Gesnerus quaedam deesse putat hunc in sensum supplenda: magis erit laudandus vel tale quid. Die restitution -ist leicht; gleichklang und buchstabenähnlichkeit verursachte den ausfall. Phavorinus der voll dichterreminiscenzen steckt gebrauchte wohl die homerische phrase: ov่xćt ảvexzós umgeformt in das prosaische $0 \dot{v} \times \varepsilon \varepsilon^{\prime} \quad \dot{\alpha} v \varepsilon \times \tau \tilde{\omega} \xi \varepsilon \varepsilon^{\prime} \chi \varepsilon \imath$.

Dels.

M. Schmidt. 